\title{
Study of Latest Trend on Acupuncture for Obesity Treatment
}

\author{
Hea-Sun Chun ${ }^{1}$, Dong-Hwan Kim ${ }^{2}$, Ho-Seub Song ${ }^{1} *$ \\ ${ }^{1}$ Department of Acupuncture \& Moxibustion Medicine, College of Korean Medicine, Gachon University, Seongnam, Republic of Korea \\ ${ }^{2}$ Department of Acupuncture \& Moxibustion Medicine, College of Korean Medicine, Kyunghee University, Seoul, Republic of Korea
}

Received September 30, 2021

Reviewed October 9, 2021

Accepted December 3, 2021

\section{*Corresponding Author}

Ho-Seub Song

Department of Acupuncture \& Moxibustion Medicine, College of Korean Medicine, Gachon University, 1342 Seongnam-daero, Sujeong-gu, Seongnam 13120, Republic of Korea Tel: +82-31-750-5424

E-mail: hssong70@gachon.ac.kr
Objectives: The aim of this review was to appraise Korean studies published between 2010 and 2021 which examined the role of acupuncture in the treatment of obesity. Methods: We performed a search of the NDSL, KISS, RISS, OASIS, PubMed, EMBASE electronic databases for relevant animal researches, case reports, and clinical trials, using the following search terms: 'obesity', 'acupuncture', 'electroacupuncture', and 'pharmacopuncture'. We excluded previous reviews and meta-analyses, studies not related to obesity or acupuncture treatment, as well as studies conducted in countries other than Korea. We also excluded studies where relevant information on acupuncture treatment in obesity could not be obtained.

Results: Most studies were conducted in animals, followed by case reports and clinical trials. In animal researches and case reports, pharmacopuncture was the most used intervention. In case studies, electroacupuncture, thread-embedding therapy, manual acupuncture, acupotomy, and auricular acupuncture were also used. In animal researches, pharmacopuncture treatment was associated with improvement in obesity indices. In the case of local obesity, specific acupuncture techniques such as thread-embedding therapy and pharmacopuncture were associated with significant improvements in local obesity, even when diet and exercise were not controlled for.

Conclusion: Acupuncture treatment showed significant benefit in the treatment of obesity, with a local effect evident for certain approaches, such thread-embedding therapy and acupotomy.

Keywords: acupuncture, local obesity, obesity treatment, review

\section{INTRODUCTION}

Obesity is a common condition in which excess fat is stored in the body due to calorie intake exceeding the energy demand for physical activity and growth. It is often associated with nutrient imbalances, leading to the accumulation of triglycerides in adipose tissue [1]. The prevalence of obesity among adults 19 years of age and older increased by 2.1\% from 2007 (31.7\%) to 2019 (33.8\%). In males obesity prevalence increased by $5.6 \%$, from $36.2 \%$ to $41.8 \%$ [2]. The need for obesity treatment is increasing, since it is often associated with high blood pressure, diabetes, and hyperlipidemia as known risk factors for cardiovascular disease and mortality [3]. The growing prevalence of obesity in Korea further motivates this need.

Several studies have examined the scope of the obesity epidemic from a Korean perspective. Kim et al. [4] examined trends in obesity research, with a particular focus on pharmacopuncture in a follow-up publication [5]. Kang et al. [6] conducted a review of Korean studies published between 2001 and October 2005 which examined the anti-inflammatory and analgesic effects as well as safety of pharmacopuncture. No studies have since performed a critical appraisal of research examining the role of acupuncture in the treatment of obesity.

The aim of this review was therefore to examine subsequent research trends in studies examining the role of acupuncture as a treatment for obesity (2010-2021). We anticipated that insight 
gathered as a result of this study could help inform future research and clinical trials.

\section{METERIALS AND METHODS}

\section{Data sources and searches}

We searched the NDSL, KISS, RISS, OASIS, PubMed and EMBASE electronic database for studies published between January 2010 and August 2021, using the following search terms: 'obesity', 'acupuncture', 'electroacupuncture', and 'pharmacopuncture'.

\section{Data selection (Fig. 1)}

The procedures used for data selection are illustrated in Fig. 1. We selected full-text studies published in domestic academic journals and dissertations, which examined acupuncture treatments in obesity, screened for review based on the publication titles. We excluded previous reviews, meta-analyses and protocols, studies which did not examine obesity or acupuncture treatment, studies published outside Korea, and studies published before 2010. We selected relevant animal researches, case reports and clinical trials for inclusion in our review. Full-text articles which lacked available data or did not focus on acupuncture as a treatment for obesity were also excluded.

Retrieved records ( $n=604$ ) (PubMed: 23 , EMBASE: 9, NDSL: 24
KISS: 111, RISS: 163, OASIS: 56 )

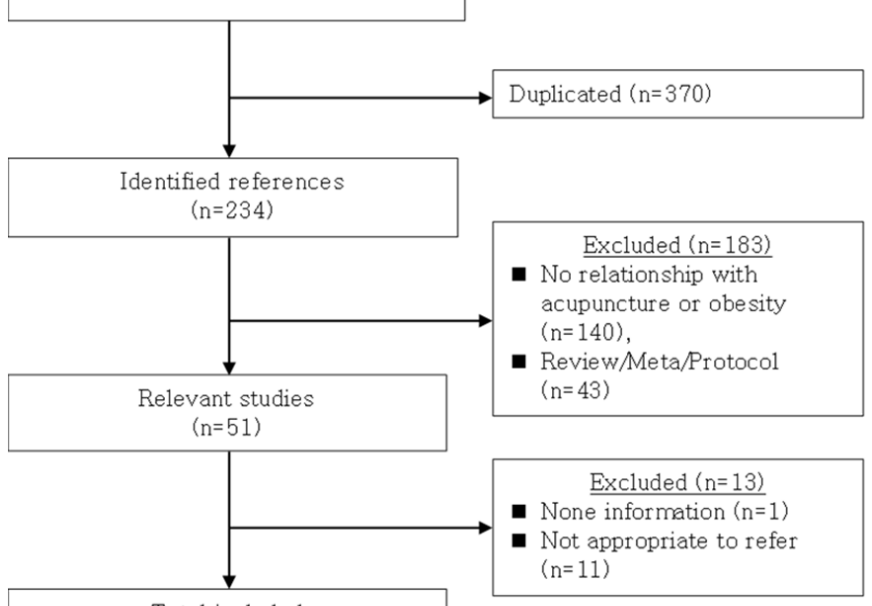

Total included $(n=38)$

Figure 1. Reference flow chart.

\section{Data analysis}

Tables and graphs were prepared using Excel 2019 (Microsoft Corp., Radmond, WA, USA). The frequencies and distributions of each item were summarized in Table format. Where necessary, the overall distribution was plotted in a graph.

\section{RESULTS}

The types of acupuncture used in the studies under examination were pharmacopuncture, electroacupuncture, threadembedding therapy, manual acupuncture, acupotomy, auricular acupuncture.

\section{Classifications of acupuncture type (Fig. 2)}

Pharmacopuncture was the most used technique (53\%), followed by electroacupuncture, auricular acupuncture, and acupotomy, as shown in Fig. 2.

\section{Summary of acupuncture type}

\section{1) Summary of acupuncture type according to research} type (Fig. 3)

Most of the studies that used pharmacopuncture were conducted in animal researches, while the use of auricular acupuncture was limited to one clinical trial (Fig. 3). In most case studies, acupuncture and other Korean medicinal treatments

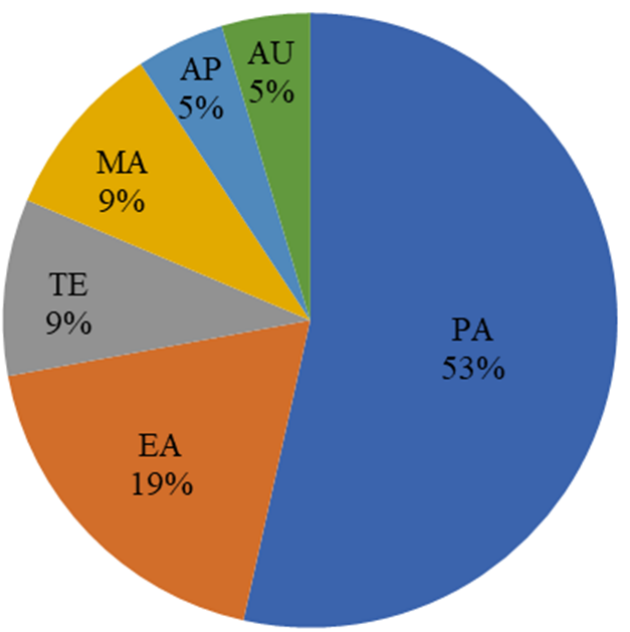

Figure 2. Classifications of acupuncture type. PA, Pharmacopuncture; MA, Manual Acupuncture; EA, Electronic Acupuncture; AP, Acupotomy; TE, Tread-Embedding; AU, Auricular Acupuncture. 


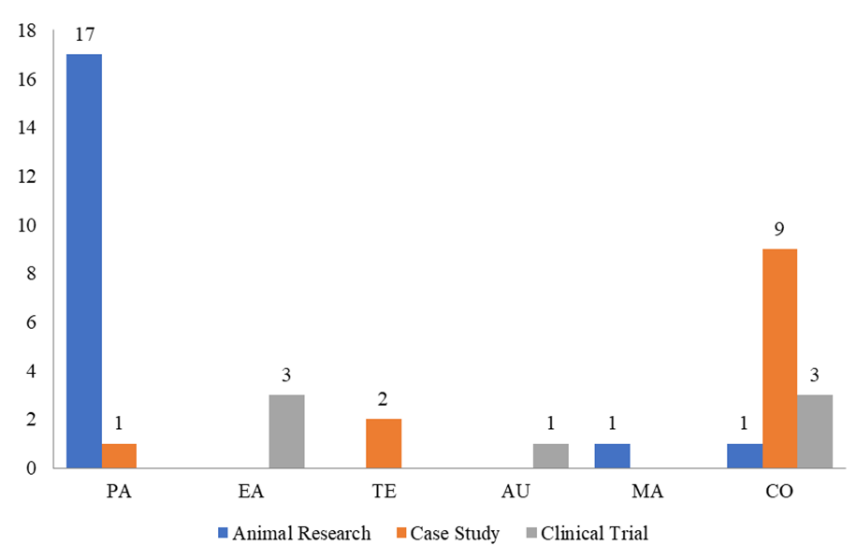

Figure 3. Summary of acupuncture type according to the research type. PA, Pharmacopuncture; MA, Manual Acupuncture; EA, Electronic Acupuncture; AP, Acupotomy; TE, Tread-Embedding; AU, Auricular Acupuncture; $\mathrm{CO}$, Combined Treatment.

were combined.

\section{2) Summary of acupuncture type according to research subjects (Fig. 4)}

Most animal researches used pharmacopuncture, while in human studies, most used combined treatment. In the case of a human research, various types of acupuncture, including electroacupuncture, auricular acupuncture, and thread-embedding therapy were used.

\section{Classifications of study (Fig. 5)}

The number of studies per classification category were: animal researches $(n=19 ; 50 \%)$, case studies $(n=12 ; 32 \%)$, and clinical trials $(\mathrm{n}=7 ; 18 \%)$, as shown in Fig. 5 .

\section{Summary of studies (Table 1)}

In the case of animal researches, the techniques used were pharmacopuncture $(n=17)$, manual acupuncture $(n=1)$, and a combination of pharmacopuncture and herbal medicine ( $\mathrm{n}$ $=1$ ). In studies involving human subjects, the majority used a combination of acupuncture and other treatments $(n=9)$, while others were limited to either thread-embedding therapy $(\mathrm{n}=2)$ or pharmacopuncture $(\mathrm{n}=1)$. In several studies, acupuncture was combined with other forms of treatment, including herbal medicines, moxibustion, and cooling therapy. In one case report [7], an obese pediatric patient was treated with a combination of electroacupuncture, herbal medicines, moxi-

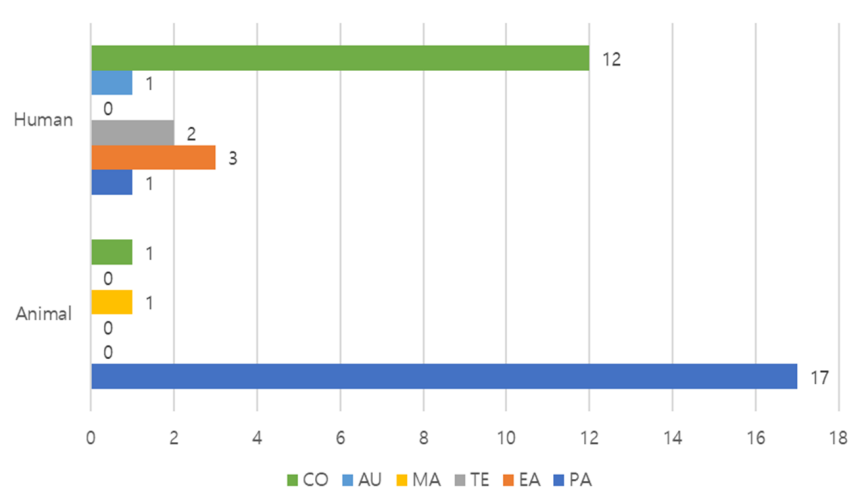

Figure 4. Summary of acupuncture type according to the research subjects. PA, Pharmacopuncture; MA, Manual Acupuncture; EA, Electronic Acupuncture; TE, Tread-Embedding; AU, Auricular Acupuncture; CO, Combined Treatment.

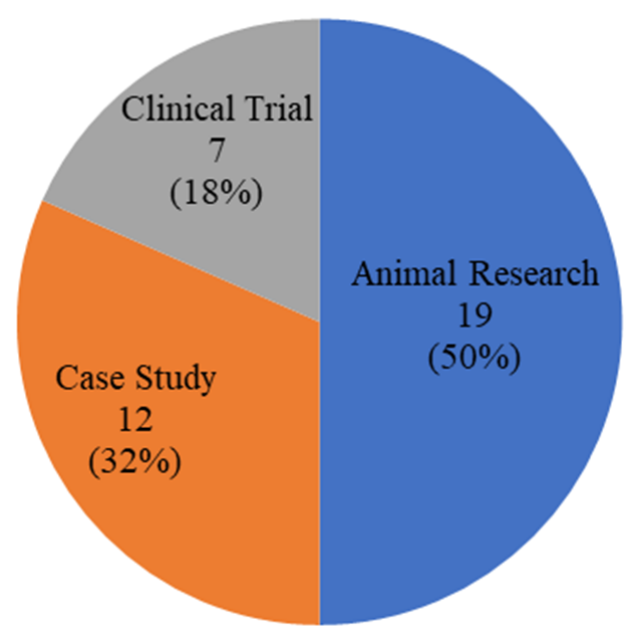

Figure 5. Classifications of study.

bustion, and auricular acupuncture. In total, three clinical trials used electroacupuncture, either alone or in combination with manual acupuncture and electroacupuncture, while one clinical trial used auricular acupuncture.

\section{Analysis by study}

\section{1) Animal researches (Table 2)}

A total of 19 animal researches were included in this review, as summarized in Table 2. The types of pharmacopuncture used were Lumbricus pharmacopuncture, Glycine Semen Germinatum pharmacopuncture, Rhizoma Atractylodis pharmacopuncture, Arecae Pericarpium pharmacopuncture, Crataegus Pinnaifida phamacopuncture, Artemisia capillaries pharmacopuncture, Rhei Radix et Rhizoma phrmacopunc- 
Hea-Sun Chun, et al.

Table 1. Summary of study

\begin{tabular}{|c|c|c|c|c|c|}
\hline Subject & Study & \multicolumn{2}{|c|}{ Acupuncture type } & $\mathrm{N}$ & $\%$ \\
\hline \multirow[t]{3}{*}{ Animal } & Animal research & PA [20-31, 33-37] & & 17 & 44 \\
\hline & & MA [38] & & 1 & 3 \\
\hline & & Combined treatment & $\mathrm{PA}+\mathrm{HM}[32]$ & 1 & 3 \\
\hline \multirow[t]{13}{*}{ Human } & Case study & TE $[41,42]$ & & 2 & 6 \\
\hline & & $\mathrm{PA}[40]$ & & 1 & 3 \\
\hline & & Combined treatment & $\mathrm{PA}+\mathrm{TE}[43,44]$ & 9 & 23 \\
\hline & & & $\mathrm{PA}+\mathrm{Moxa}[48]$ & & \\
\hline & & & $\mathrm{PA}+\mathrm{HS}[49]$ & & \\
\hline & & & $\mathrm{AP}+\mathrm{CT}[45]$ & & \\
\hline & & & $\mathrm{AP}+\mathrm{CT}+\mathrm{HM}[46]$ & & \\
\hline & & & $\mathrm{EA}+\mathrm{HM}[39]$ & & \\
\hline & & & $\mathrm{EA}+\mathrm{AU}+\mathrm{Moxa}+\mathrm{HM}[7]$ & & \\
\hline & & & $\mathrm{MA}+\mathrm{EA}[47]$ & & \\
\hline & Clinical trial & EA [16-18] & & 3 & 7.5 \\
\hline & & $\mathrm{AU}[19]$ & & 1 & 3 \\
\hline & & Combined treatment & $\mathrm{MA}+\mathrm{EA}[8,14,15]$ & 3 & 7.5 \\
\hline- & Total & - & & 38 & 100 \\
\hline
\end{tabular}

PA, Pharmacopuncture; HM, Herbal Medicine; TE, Thread-Embedding; HS, Heat Stimulus; CT, Cold Therapy; EA, Electroacupuncture; AU, Auricular Acupuncture; Moxa, Moxibustion; MA, Manual Acupuncture; AP, Acupotomy.

ture, Wild Ginseng Complex pharmacopuncture, Eugenia caryophyllata Thumb pharmacopuncture, Eriobotryae Folium pharmacopuncture, Curcume Radix pharmacopuncture, Pericarpium Zanthoxyli pharmacopuncture, Ephedrasinica StaphAconitum carmichaeli Debeaux pharmacopuncture, Cultivated Wild Ginseng pharmacopuncture, Cultivated Ginseng pharmacopuncture, Red Ginseng pharmacopuncture, Ephedra sinica pharmacopuncture, Evodiae Fructus pharmacopuncture, Nelumbo nucifera pharmacopuncture, Pinellia ternate pharmacopuncture, and Taraxacum platycarpum pharmacopuncture. Also, there was a study comparing the anti-obesity effect according to each acupoins by inserting acupuncture into two acupoints. The acupoints used were: CV12, ST36, CV13, BL23, ST25, BL21, BL20, LR14, HT7, and BL13.

\section{2) Case study (Table 3)}

The present review included a total of 12 case studies, which are summarized in Table 3. The treatment modalities used included manual acupuncture, pharmacopuncture, electroacupuncture, thread-embedding therapy, acupotomy, auricular acupuncture, and moxibustion, among others. Several case studies, focused on obesity localized to the abdomen, forearms, or thighs. Most of the studies focused on the treatment of localized obesity did not control diet and exercise. The acupoints used were: ST25, ST36, ST21, ST27, ST28, ST38, ST40, ST44, CV5, CV4, CV3, CV8, CV6, CV9, CV10, CV12, BL36, BL57, BL58, BL59, SP15, SP14, LI4, LI11, and LR3.

\section{3) Clinical trials (Table 4)}

A total of seven clinical trials were included in the present review, as summarized in Table 4. In studies where electroacupuncture was used, most of the patients had abdominal obesity. In total, two clinical trials compared the effects of combined manual acupuncture and electroacupuncture with the sham acupuncture group. Kim et al. [8] analyzed the effects of acupuncture on obesity through analysis of heart rate variability and InLF/HF after manual acupuncture and electroacupuncture treatment. There was one study using auricular acupuncture. The acupoints used were: CV12, CV6, SP6, SP15, SP14, LI4, LI11, ST25, ST27, ST28, ST36, and ST44

\section{DISCUSSION}

Due to recent dietary changes, obesity is increasing due to an 
Table 2. Classifications of animal research

\begin{tabular}{|c|c|c|c|}
\hline Study/Year & Treatment & Injections & Acupoint/Injection Point \\
\hline Kwon (2010) [29] & Pharmacopuncture & Lumbricus & CV12, ST36 \\
\hline Park (2010) [20] & Pharmacopuncture & Glycine Semen Germinatum & CV13 \\
\hline Youh (2010) [25] & Pharmacopuncture & Rhizoma Atractylodis & BL23 \\
\hline Lee (2010) [21] & Pharmacopuncture & Arecae Pericarpium & ST25 \\
\hline Youh (2011) [26] & Pharmacopuncture & Artemisia capillaries & BL20 \\
\hline Ryu (2012) [24] & Pharmacopuncture & Rhei Radix et Rhizoma & BL20 \\
\hline Kim (2012) [33] & Pharmacopuncture & Wild Ginseng Complex & BL23 \\
\hline Kim (2013) [27] & Pharmacopuncture & Eugenia caryophyllata $T_{\text {HUNB. }}$ & Intraperitoneal/Subcutaneous \\
\hline Roh (2013) [28] & Pharmacopuncture & Eriobotryae Folium & Intraperitoneal/Subcutaneous \\
\hline Ryu (2013) [23] & Pharmacopuncture & Curcume Radix & LR14 \\
\hline Kim (2014) [34] & Pharmacopuncture & $\begin{array}{l}\text { Pericarpium Zanthixyli, Ephedra } \\
\text { sinica Staph Aconitum carmichaeli } \\
\text { Debeaux and Cultivated Wild Ginseng }\end{array}$ & BL20 \\
\hline Koo (2016) [35] & Pharmacopuncture & $\begin{array}{l}\text { Cultivated Ginseng, Wild Ginseng, } \\
\text { Red Ginseng }\end{array}$ & BL13 \\
\hline $\operatorname{Kim}(2016)[30]$ & Pharmacopuncture & Ephedra sinica & Subcutaneous \\
\hline Seol (2016) [31] & Pharmacopuncture & Evodiae fructus & Subcutaneous \\
\hline Cheong (2017) [22] & Pharmacopuncture & Nelumbo nucifera & CV12 \\
\hline Lee (2020) [36] & Pharmacopuncture & Pinellia ternata & Inguinal fat \\
\hline Nam (2021) [37] & Pharmacopuncture & Taraxacum platycarpum & Inguinal fat \\
\hline Woo (2014) [38] & Manual Acupuncture & - & HT7, ST36 \\
\hline \multicolumn{4}{|l|}{ Combined treatment } \\
\hline Bang (2011) [32] & $\begin{array}{l}\text { Pharamcopuncture/ } \\
\text { Herbal Medicine }\end{array}$ & Crataegus pinnaifida & BL21 \\
\hline
\end{tabular}

increase in high-calorie and high-fat intake, excessive drinking, and lack of exercise [9], obesity is a problem because it is closely related to chronic diseases such as diabetes, hyperlipidemia, high blood pressure, coronary artery disease and stroke [10].

Currently, for the treatment of obesity, lifestyle modification methods such as diet, exercise therapy, and behavioral therapy, drug therapy, and surgical treatment are being implemented, drugs such as appetite suppressant(Reductil, ingredient name Sibutramine) and fat digestion inhibitor(Xenical, ingredient name Orlistat) have various side effects and disadvantages due to contraindications for use [11].

In Korean Medicine, doctors thought of the causes of obesity as deficiency syndrome like deficiency of Qi and yang of spleen and kidney, and like sitting down for a long time and lying down for a long time and as excess syndrome like high-calorie \& high-fat intake, phlegm, spleen-stomach heat stasis, and blood stasis. In addition, as the treatment method, it suggested a method of improving spleen function, improving deficiency of Qi, and warming in the case of deficiency syndrome, and Dampness phlegm-Dispelling, Water Draining, Digesting, and circulating blood and extinguishing blood stasis method in the case of excess syndrome [12].

Korean Medicine treatment for obesity includes diet and exercise therapy, along with herbal medicine therapy, auricular acupuncture, electroacupuncture, manual acupuncture, cupping therapy, bowel cleansing therapy, Qigong and meditation therapy [13].

Recently, various studies and clinical papers using acupuncture in obesity have been published, and the domestic research trend of obesity using acupuncture was analyzed to be helpful in future obesity treatment and research.

When analyzing the latest trends in acupuncture treatment for obesity, the types of acupuncture used were manual acupuncture, electroacupuncture, pharmacopuncture, threadembedding therapy, acupotomy, and auricular acupuncture.

In the case of clinical trials, significant improvement of fac- 
Hea-Sun Chun, et al.

Table 3. Classifications of case study

\begin{tabular}{|c|c|c|c|c|}
\hline Study/Year & Treatment & $\mathrm{N}$ & Obesity & Acupoint/Injection point \\
\hline Go (2015) [39] & $\mathrm{EA}$ & 3 & Obesity & Thigh area \\
\hline Shin (2011) [41] & TE & 9 & Localized obesity & Forearm, Thigh, Abdominal \\
\hline Shin (2012) [42] & $\mathrm{TE}$ & 5 & Localized obesity & Forearm, Thigh, Abdominal \\
\hline Hwang (2018) [40] & $\mathrm{PA}$ & 2 & Localized obesity & Abdominal area \\
\hline \multicolumn{5}{|l|}{ Combined treatment } \\
\hline Shin (2013) [43] & PA/TE & 5 & Abdominal obesity & ST25, CV5, CV4, CV3 (PP); ST25-CV8 (TE) \\
\hline Chon (2015) [44] & $\mathrm{PA} / \mathrm{TE}$ & 5 & Localized obesity & $\begin{array}{l}\text { ST25, CV8, CV6, CV4, CV3, CV9, CV12, } \\
\text { BL36, BL57, BL58, BL59 }\end{array}$ \\
\hline Yun (2015) [47] & $\mathrm{MA} / \mathrm{EA}$ & 6 & Abdominal obesity & $\begin{array}{l}\text { CV12, CV6, ST25, ST21, SP15, SP14, CV8, LI4, LI11, } \\
\text { ST36, ST44 (MA); ST21, SP14, ST25, CV8 (EA) }\end{array}$ \\
\hline Cho (2017) [46] & $\mathrm{AP} / \mathrm{CT} / \mathrm{HM}$ & 24 & Localized obesity & Subcutaneous fat \\
\hline Yoon (2015) [45] & $\mathrm{AP} / \mathrm{CT}$ & 4 & Localized obesity & Subcutaneous fat \\
\hline Park (2016) [48] & PA/Moxa & 5 & Abdominal obesity & CV10, CV4, ST25, ST27, SP15, SP14 \\
\hline Yoo (2016) [49] & $\mathrm{PA} / \mathrm{HS}$ & 2 & $\begin{array}{l}\text { Abdominal obesity } \\
\text { (woman) }\end{array}$ & CV6, CV4, ST25, ST28, SP15, SP14 \\
\hline Jung (2011) [7] & $\mathrm{EA} / \mathrm{AU} / \mathrm{Moxa} / \mathrm{HM}$ & 1 & Child obesity & $\begin{array}{l}\text { Abdominal, LI4, LI11, ST36, ST38, ST40, LR3 (EA); } \\
\text { CV12, CV4 (MOXA); Shenmen, Spleen, Stomach, } \\
\text { Endocrine, Hunger point (AU) }\end{array}$ \\
\hline
\end{tabular}

PA, Pharmacopuncture; TE, Thread-Embedding; MA, Manual Acupuncture; EA, Electroacupuncture; AU, Auricular Acupuncture; AP, Acupotomy; CT, Cold Therapy; HM, Herbal Medicine; Moxa, Moxibustion; HS, Heat Stimulus.

Table 4. Classifications of clinical trial

\begin{tabular}{|c|c|c|c|c|}
\hline Study/Year & Treatment & $\mathrm{N}$ (group) & Obesity & Acupoint/Injection point \\
\hline Song (2010) [16] & EA & 10 & Abdominal obesity & Abdominal, Subcutaneous fat \\
\hline Chung (2010) [17] & EA & $\begin{array}{l}35 \text { (EA group 12, sham EA group } \\
\text { 11, waitlist group 12) }\end{array}$ & Abdominal obesity & $\begin{array}{l}\text { CV12, CV6, ST25, SP15, SP14 } \\
\text { LI4, LI11, ST36, ST44 }\end{array}$ \\
\hline Oh (2017) [18] & EA & $\begin{array}{l}12 \text { (Insulated needle group 5, } \\
\text { control group 7) }\end{array}$ & Abdominal obesity & CV12, CV6, ST25, ST27, SP15 \\
\hline Yeo (2014) [19] & $\mathrm{AU}$ & $\begin{array}{l}58 \text { (Five AU group 22, Hunger AU } \\
\quad \text { group 21, Sham control group 15) }\end{array}$ & Overweight & $\begin{array}{l}\text { Shenmen, Spleen, Stomach, } \\
\text { Hunger, Endocrine }\end{array}$ \\
\hline $\operatorname{Kim}(2016)[8]$ & $\mathrm{MA} / \mathrm{EA}$ & 37 & Obesity (woman) & $\begin{array}{l}\text { LI4, LI11, SP6, ST36, CV12, } \\
\text { CV6 (MA); ST25, ST28 (EA) }\end{array}$ \\
\hline Shin (2018) [14] & $\mathrm{MA} / \mathrm{EA}$ & $\begin{array}{l}91 \text { (Acupuncture group 48, Sham- } \\
\text { acupuncture control group 43) }\end{array}$ & Obesity (woman) & $\begin{array}{l}\text { LI4, LI11, SP6, ST36, CV6, } \\
\text { CV12 (MA); ST25, ST28 (EA) }\end{array}$ \\
\hline $\operatorname{Kim}(2021)[15]$ & $\mathrm{MA} / \mathrm{EA}$ & $\begin{array}{l}91 \text { (Treatment group 48, Control } \\
\text { group 43) }\end{array}$ & Obesity (woman) & $\begin{array}{l}\text { LI4, LI11, SP6, ST36, CV6, } \\
\text { CV12 (MA); ST25, ST28 (EA) }\end{array}$ \\
\hline
\end{tabular}

MA, Manual Acupuncture; EA, Electroacupuncture; AU, Auricular Acupuncture.

tors related to obesity was found in the group treated with acupuncture.

When manual acupuncture + electroacupuncture is applied to the acupoint, certain carnitine (L2, L4, L6 and L-Carnitine) levels significantly increased, compared to the sham acupuncture group $[14,15]$.
When electroacupuncture was inserted into the abdominal subcutaneous fat layer, improvement of obesity index such as WC (waist circumference) etc. was confirmed [16-18].

In case of auricular acupuncture, there was a significant decrease in BMI in the auricular acupuncture group [19].

In the case of animal researches, pharmacopuncture was 
used the most, and the types of pharmacopuncture and the acupoint was various, and the improvement and stability of obesity indicators were confirmed.

A decrease in dietary intake [20-22] and a decrease in dietary efficiency $[21,23]$ were confirmed. Especially, Ryu et al. [24] showed a marginal decrease in weight loss and dietary efficiency.

The effects of a decrease in weight gain and a decrease in adipose tissue proliferation $[25,26]$ were confirmed. In addition, there were studies confirming a decrease in adipocyte size and a decrease in plasma Triglycerides and Total-Cholesterol $[27,28]$. Kwon and Park [29] confirmed the antioxidant, proinflammatory and lipid lowering effects including Triglycerides and Total-Cholesterol.

Kim et al. [30], Seol [31] confirmed the effect on obesity by confirming the decrease in the weight of subcutaneous fat.

There were also studies confirming a decrease in leptin [32$35]$ and the regulation of lipolytic metabolism [36, 37].

The reason that pharmacopuncture was most often used in animal researches may be due to the condition of being a laboratory rat, pharmacopuncture can prevent the drug from being destroyed in the gastrointestinal tract and can be used for patients who are difficult to swallow [29], the combination of effective exercise therapy and diet therapy for obesity is difficult to apply to modern life, so it has been used in many ways based on advantages such as obesity treatment using pharmacopuncture as one of the effective treatment options [27].

In the case of animal researches using manual acupuncture, it was confirmed that the factors related to obesity were affected by the acupoint [38].

In case studies, combination treatment was more common than single treatment, and various types of acupuncture were used. Acupuncture treatment showed significant improvement in obesity index in clinical practice, but there was a limitation in that most cases were combined treatment.

In the case of single treatment, there was an improvement in the index of local obesity $[39,40]$, however, in the case of thread-embedding therapy, the limitations of thread-embedding therapy alone treatment were also pointed out $[41,42]$.

In the case of combined treatment, significant improvement in obesity index was observed, but there is a point that it is not known which treatment resulted in improvement.

Thread-embedding therapy was mainly used for the treatment of local obesity in case studies. Thread-embedding therapy showed improvement in local obesity in parallel with pharmacopuncture $[43,44]$.

Acupotomy was usually performed with cryolysis, there was an improvement in the local obesity index in the treated area despite weight gain [45].

As such, animal researches were conducted using pharmacopuncture in most cases, and there was an improvement in obesity index and in some researches reported there was no hepatotoxicity $[21,28,33]$. So it is judged to be the basis for efficacy and safety in clinical obesity treatment. And clinical trial and case study showed acupuncture treatment showed a significant effect in the treatment of obesity, and in particular, electroacupuncture, pharmacopuncture, thread-embedding therapy, and acupotomy were shown to be effective in treating local obesity. In addition, although there are various side effects and disadvantages due to contraindications for use of the existing Western medicine [11], as a result of analyzing researches of obesity in Korean medicine for 10 years, one case of side effect was reported. It was an epidermal frostbite that occurred during cryolysis, and it recovered after 2 weeks, leaving no scars [46]. However, case studies have a limitation in that most cases are combined treatment rather than acupuncture treatment alone.

And considering the relatively small number of studies in the case of acupotomy and auricular acupuncture and the small number of cases studies, we thought that active research on Korean medicine obesity treatment is needed in the future.

\section{CONCLUSIONS}

In conclusion, we conducted a review of animal researches, case reports and clinical trials which examined the role of acupuncture as a treatment for obesity. Most animal researches (53\%) used pharmacopuncture, while case studies involved multiple methods, including acupotomy, thread-embedding therapy, and electroacupuncture, among others. First, findings from animal researches suggested that pharmacopuncture improves obesity indices. Second, case studies and clinical trials conducted in human participants suggested the value of acupuncture as a treatment for obesity in the Korean population. Certain forms of acupuncture, such as thread-embedding therapy and pharmacopuncture, appeared to have value in the treatment of localized obesity, even when diet and exercise were not controlled for. 


\section{CONFLICTS OF INTEREST}

The authors declare no conflict of interest.

\section{ORCID}

Hea-Sun Chun, https://orcid.org/0000-0002-1002-9312

Dong-Hwan Kim, https://orcid.org/0000-0001-5734-2181

Ho-Seub Song, https://orcid.org/0000-0001-5306-8795

\section{REFERENCES}

1. Department of Rehabilitation Medicine, National College of Oriental Medicine. DongEui rehabilitation medicine. Seoul: SeoWonDang; 1995. p. 570-3.

2. Noncommunicable Disease (NCD) Statistics [Internet]. Cheongju: Division of Chronic Disease Control, Korea Disease Control and Prevention Agency; c2021 [cited 2021 Oct 7]. Available from: https://www.kdca.go.kr/board/board.es?mid=a206020100 00\&bid=0034\&act=view\&list_no=717196\#.

3. Park HS, Cho HJ, Kim YS, Kim CJ. The diseases associated with obesity in Korean adults. J Korean Acad Fam Med. 1992;13(4):344-53.

4. Kim DH, Shin WS, Kim DH, Jeong YJ, Im HB, Park WH, et al. An analysis of domestic medicine study tendency on obesityfocused on The Korean Journal of Obesity. J Korean Med Obes Res. 2013;13(1):1-9.

5. Kim MW, Song YK, Lim HH. Study of experimentations and clinical trials' trends for obesity treatment using pharmacupuncture. J Soc Korean Med Obes Res. 2011;11(1):47-60.

6. Kang JH, Heo DS, Yoon IJ, Oh MS. An analysis of tendencies of studies on herbal acupuncture - focusing on domestic theses since 2001 about anti-inflammation, pain relief and anti-obesity effects, including safety. J Korean Orient Med. 2007;28(2):93113.

7. Jung SK, Yu SA, Lee SY. A case report about obese child. J Korean Orient Pediatr. 2011;25(2):94-101.

8. Kim JI, Yang YC, Kim KW, Cho JH, Kim SY, Park HJ, et al. The correlation analysis between heart rate variability and effect of acupuncture on obese women. J Korean Med Rehabil. 2016;26(4):85-96.

9. Department of Internal Medicine, Seoul National University College of Medicine. Update in internal medicine. Seoul: Gunja Publishing; 1997. 852 p.

10. Lee JH. Treatment of obesity. J Korean Soc Study Obes. $1992 ; 1(1): 21-4$

11. Kim CH. Pharmacologic therapy for obesity. Korean Clin Dia- betes. $2008 ; 9(3): 173-7$

12. Lee BJ, Kim SH. The study of record relate with the notion, the diagnosis and treatment for obesity. Daejeon Univ Inst Orient Med. 1998;7(1):533-41.

13. The Society of Korean Medicine Rehabilitation. Oriental rehabilitation medicine. Seoul: Gunja Publishing; 2003. p. 349-50.

14. Shin WC. Effects of acupuncture on serum metabolic parameters in premenopausal obese women [master's thesis]. [Seoul]: Kyung Hee University; 2018.

15. Kim KW, Shin WC, Choi MS, Cho JH, Park HJ, Yoo HH, et al. Effects of acupuncture on anthropometric and serum metabolic parameters in premenopausal overweight and obese women: a randomized, patient- and assessor-blind, sham-controlled clinical trial. Acupunct Med. 2021;39(1):30-40.

16. Song SM, Song YK, Lim HH. The effect of electroacupuncture on abdominal fat deposit and parameters for obesity. J Oriental Rehab Med. 2010;20(2):113-27.

17. Chung JY, Kim JI, Lee SH, Kang SK. Effects of electroacupuncture on parameters related to obesity in adults with abdominal obesity: three arm randomized single blind pilot study. J Korean Acupunct Moxib Soc. 2010;27(6):43-57.

18. Oh SY, Lee H, Yun GW, Kang JH. Efficacy of electroacupuncture using an insulated needle in adults with abdominal obesity: a pilot study. Acupuncture. 2017;34(2):49-59.

19. Yeo S, Kim KS, Lim S. Randomised clinical trial of five ear acupuncture points for the treatment of overweight people. Acupunct Med. 2014;32(2):132-8.

20. Park JE. The effects of Glycine Semen Germinatum herbal acupuncture at Sangwan(CV13) on the obese rats induced by high fat diet [master's thesis]. [Naju]: DongShin University; 2010.

21. Lee JE. The effects of Arecae Pericarpium pharmacopunture at Cheon-chu(ST25) on the obese rats induced by high fat diet [dissertation]. [Naju]: DongShin University; 2010.

22. Cheong MS. The effect of nelumbo nucifera pharmacopuncture at Jung-wan (CV12) on the obese rats induced by high fat diet [master's thesis]. [Naju]: DongShin University; 2017.

23. Ryu HS. The effects of Curcume Radix pharmacopuncture at Qimen(LR14) on the lipid lowering, anti-oxidative activity and concentration of proinflammatory cytokines in obese rats fed high fat diet [dissertation]. [Naju]: DongShin University; 2013.

24. Ryu HS, Kim SP, Kim SM, Lee DG, Lee OJ, Lee JH, et al. The effects of Rhei Radix et Rhizoma pharmacopuncture at Bi$s u$ (BL20) on the obese rats induced by high fat diet. J Korean Acupunct Moxib Med Soc. 2012;29(3):9-18.

25. Youh EJ, Seo BK, Nam SS, Kang SK. Anti-obesity effect of rhizoma atractylodis herbal acupuncture in high fat diet-induced obese ICR mouse. J Korean Acupunct Moxib Soc. 2010;27(6):3142. 
26. Youh EJ, Seo BK, Huang B, Kim JI, Kang SK. Artemisia capillaries herbal acupuncture improves metabolic abnormalities in high fat diet-induced obese ICR mice. J Korean Acupunct Moxib Soc. 2011;28(1):1-14.

27. Kim SJ, Lee HJ, Lee BH, Lee YK, Lim SC, Jung TY, et al. The effects of pharmacopuncture(Eugenia caryophyllata ${ }_{\text {THUNB. }}$.) on the high fat diet-induced obese ICR mice. Acupuncture. 2013;30(3):75-85.

28. Roh SS, Kim JS, Yim SC. The efficacy of Eriobotryae Folium pharmacopuncture on local fat of high fat diet induced obesity mice. Acupuncture. 2013;30(3):15-25.

29. Kwon K, Park HS. The effects of lumbricus pharmacopuncture on the lipid lowering, anti-oxidative activity and concentration of proinflammatory cytokines in rat fed high fat diet. J Korean Acupunct Moxib Soc. 2010;27(2):59-69.

30. Kim HJ, Kim EJ, Han YH. The effect of Ephedra sinica pharmacopuncture on lipid metabolism in an experimental mouse model of obesity. J Int Korean Med. 2016;37(4):579-90.

31. Seol MH. The lipolytic and related mechanism of evodiae fructus on obese mouse model induced by high fat diet [master's thesis]. [Seoul]: Kyung Hee University; 2016.

32. Bang SP. Effects of Crataegus pinnaifida phamacopuncture at $\mathrm{Wi}$-su(BL21) and Taeumchowitang on obese rats [dissertation]. [Naju]: DongShin University; 2011.

33. Kim MW, Lim HH, Song YK. Anti-obesity effect of wild ginseng complex pharmacopuncture on adipocyte and high fat diet-induced obese C57BL/6J mice. J Oriental Rehab Med. 2012;22(2):67-90.

34. Kim SH. Inhibitory effects of Pericarpium zanthoxyli, Ephedra sinica staph - Aconitum carmichaeli debeaux and cultivated wild ginseng pharmacopuncture on obesity induced by high fat diet in mice [dissertation]. [Wanju]: Woosuk University; 2014.

35. Koo JS. Effects of cultivated ginseng, wild ginseng, red ginseng pharmacopuncture on anti-obesity in high fat diet-induced obese mice [master's thesis]. [Iksan]: WonKwang University; 2016.

36. Lee HS. Therapeutic effects of Pinellia ternata pharmacopuncture on lipid droplet catabolism [dissertation]. [Seoul]: Kyung Hee University; 2020.

37. Nam YK, Park SJ, Kim MH, Choi Y, Yang WM. Pharmacopuncture of Taraxacum platycarpum extract reduces localized fat by regulating the lipolytic pathway. Biomed Pharmacother. 2021;141:111905.

38. Woo CH, Lee YE, Roh SS, Kim JS. Acupuncture effects of fatty mice induced by high fat diet. J Korean Med Rehabil. 2014;24(3):1-9.

39. Go NG, Lee YH, Min DL. Korean medical obesity treatments on localized fat in three Japanese. J Korean Med Obes Res. 2015;15(1):51-4

40. Hwang JH, Jung HW. Effects of pharmacopuncture with wild ginseng complex in 2 elderly patients with obesity: case report. Medicine (Baltimore). 2018;97(28):e11534.

41. Shin HY, Kwon HJ, Lee YK, Lim SC, Jung TY, Lee BH, et al. The effect of thread-embedding therapy on 9 patients with partial obesity. J Korean Acupunct Moxib Soc. 2011;28(6):27-34.

42. Shin HY, Lim SC, Lee YK, Kwon HJ, Jung TY, Lee BH, et al. The duplicate effect of thread-embedding therapy on 5 patients with obesity. J Korean Acupunct Moxib Soc. 2012;29(1):61-6.

43. Shin MS. A case series: the effects of cultivated wild ginseng pharmacopuncture and thread implantation therapy on abdominal obesity. J Korean Med Obes Res. 2013;13(1):46-50.

44. Chon YJ, Yoo JE. A case report for the effects of pharmacopuncture combined thread implantation therapy to improve localized obesity. J Korean Med Obes Res. 2015;15(2):144-8.

45. Yoon SH, Mun YJ, Cho KH. Effect of acupotomy with selective cryolysis for localized fat: case report. J Korean Med Obes Res. 2015;15(2):149-52.

46. Cho KH, Yun YH, Kim BT, Kim YS, Choi YY, Yoon SH. Effects of acupotomy and selective cryolysis including herbal medicine treatment on thigh circumference: case series. J Korean Med Obes Res. 2017;17(2):133-9.

47. Yun GW, Lee H, Kim YJ, Kang JH. A report on 6 cases of abdominal obesity using electroacupuncture combined with magnetic acupuncture. Acupuncture. 2015;32(4):213-26.

48. Park SA, Lee HJ, Baek JY, Son KW, Lim KT. The effects of Ganoderma Lucidum pharmacopuncture and moxibustion (Wangtteum) on abdominal obesity: case report. J Korean Med Obes Res. 2016;16(1):64-9.

49. Yoo JE. The effects of wild ginseng complex pharmacopuncture combined with hyperthermia on abdominal obesity in post-menopause women: case report. J Korean Med Obes Res. 2016;16(2):133-7. 